\title{
Hookworm Infection: A Neglected Cause of Overt Obscure Gastrointestinal Bleeding
}

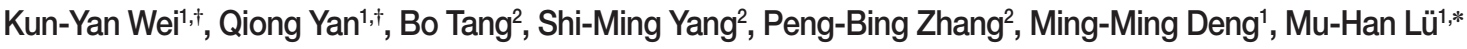 \\ ${ }^{1}$ Department of Gastroenterology, The Affiliated Hospital of Southwest Medical University, Sichuan 646000, P.R. China; \\ ${ }^{2}$ Department of Gastroenterology, Xinqiao Hospital, Third Military Medical University, Chongqing 400037, China
}

\begin{abstract}
Hookworm infections are widely prevalent in tropical and subtropical areas, especially in low income regions. In the body, hookworms parasitize the proximal small intestine, leading to chronic intestinal hemorrhage and iron deficiency anemia. Occasionally, hookworms can cause overt gastrointestinal bleeding, but this is often ignored in heavily burdened individuals from endemic infectious areas. A total of 424 patients with overt obscure gastrointestinal bleeding were diagnosed by numerous blood tests or stool examinations as well as esophagogastroduodenoscopy, colonoscopy, capsule endoscopy or double-balloon enteroscopy. All of the patients lived in hookworm endemic areas and were not screened for hookworm infection using sensitive tests before the final diagnosis. The patients recovered after albendazole treatment, blood transfusion, and iron replacement, and none of the patients experienced recurrent bleeding in the follow-up. All the 31 patients were diagnosed with hookworm infections without other concomitant bleeding lesions, a rate of $7.3 \%$ (31/424). Seventeen out of 227 patients were diagnosed with hookworm infections in the capsule endoscopy (CE), and 14 out of 197 patients were diagnosed with hookworm infections in the double balloon enteroscopy (DBE). Hookworm infections can cause overt gastrointestinal bleeding and should be screened in patients with overt obscure gastrointestinal bleeding (OGIB) in endemic infectious areas with sensitive methods. Specifically, the examination of stool specimens is clinically warranted for most patients, and the proper examination for stool eggs relies on staff's communication.
\end{abstract}

Key words: Ancylostoma duodenale, Necator americanus, hookworm, obscure gastrointestinal bleeding, double balloon enteroscopy, capsule endoscopy

\section{INTRODUCTION}

Obscure gastrointestinal bleeding (OGIB) refers to overt or occult bleeding arising from unknown reasons that persists or recurs after an initial negative examination, such as esophagogastroduodenoscopy (EGD), colonoscopy, or radiologic evaluation of the small bowel. Overt OGIB presents visible gastrointestinal (GI) bleeding with ongoing melena or hematochezia, both of which are more urgent than occult bleeding [1] and thus represent a diagnostic and therapeutic challenge for the general internist. With the development of the diagnostic method, the main modalities that have been implemented to address OGIB include double balloon enteroscopy (DBE), capsule endoscopy (CE), angiography, nuclear scans, and intraoperative enteroscopy [1]. Accumulated evidence has suggested

• Received 28 May 2017, revised 18 June 2017, accepted 23 June 2017.

*Corresponding author (52721759@qq.com)

† Kun-Yan Wei and Qiong Yan contributed equally to this study.

(c) 2017, Korean Society for Parasitology and Tropical Medicine

This is an Open Access article distributed under the terms of the Creative Commons Attribution Non-Commercial License (http://creativecommons.org/licenses/by-nc/4.0) which permits unrestricted non-commercial use, distribution, and reproduction in any medium, provided the original work is properly cited. that older patients ( $>50$ years old) are prone to bleed from angiectasias, leiomyoma, lymphoma, and adenocarcinoma, while Meckel's diverticulum and Dieulafoy's lesions are the major causes of this disorder in younger patients. Inflammatory bowel disease and nonsteroidal anti-inflammatory drug-induced small bowel disease are also causes of OGIB [2].

In developing countries located in Asia, hookworm infections, most commonly due to Ancylostoma duodenale and Necator americanus, are also an important precursor of OGIB [3], and the severity of the morbidity associated with the hookworm burden differs, ranging from mild, transient clinical signs to severe symptoms [4]. In the traditional view, hookworm disease is primarily considered to cause chronic occult blood loss leading to iron-deficiency anemia due to their attachment to the intestinal mucosa, where they chemically and mechanically rupture capillaries [4]. Therefore, the diagnostic value of hookworm infection in overt OGIB is often ignored, leading to unnecessary excessive medical tests and treatments.

Nevertheless, there is increasing awareness that more research from impoverished rural areas is needed to deepen our understanding of the etiology of the overt OGIB [5]. Over the past 
few decades, the total number of hookworm infections has increased due to the growth of the worldwide population, although environment and socioeconomic status represent the 2 most important factors impacting hookworm prevalence. Because the infection levels in areas of rural poverty are much higher, it is essential to screen all OGIB patients for all potentially endemic parasites. In China, EGD and colonoscopy are often considered as the first line of investigation to be taken in OGIB [4]. If they fail to identify the cause, additional tests are required, and some severe bleeding cases may proceed to exploratory laparotomy [4]. However, stool microscopy is not routinely undertaken in patients with acute and visible hemorrhages. Only rarely are patients examined repeatedly for hookworm eggs, as this is often impractical in large, complex centers with busy physicians and laboratories [5]. A positive result relies on communication between the physician and the laboratory. Moreover, a specific examination of the stool specimen is required for all potentially infected patients.

The objective of this study was to retrospectively assess the influence of the hookworm efforts on the incidence of overt OGIB in the southwest region of China to identify potential culprits hindering the rational investigative procedures. Preventing these delaying factors should contribute to the development of a diagnostic algorithm that will better define the choice of management strategies for these patients.

\section{MATERIALS AND METHODS}

\section{Ethical statement}

This study was approved by the Institutional Review Board of Third Military Medical University. Written informed consent was obtained from adult patients and guardians on behalf of the children enrolled in this study.

\section{Patients and methods}

We designed a descriptive retrospective study to assess the pathologic findings of 424 patients with overt OGIB collected from August 2008 to October 2016 in the affiliated hospital of southwest medical university, Sichuan and Xinqiao hospital, Third Military Medical University, Chongqing, China. Most of the patients were from the Chongqing Municipality, the Sichuan province, and the Yunnan province in the southwest region of China. Some sites in these provinces were identified as poor rural areas that might have high rates of hookworm infection. In total, we investigated 237 men and 187 women with an overall mean age of 52 years (range; $12-87$ years old).

All of the cases presented as hematemesis, intermittent melena, or hematochezia without an identified reason after numerous blood tests, EGD, colonoscopy and radiologic evaluation. In our department, patients with overt ongoing OGIB bleeding first undergo DBE or $\mathrm{CE}$, which rely on consultations among themselves and their referring doctors after the initial examination. During the study period, 227 patients underwent capsule endoscopy, and 197 underwent double-balloon enteroscopy. Capsule endoscopy was performed with an OMOM capsule (Chongqing Jinshan Science \& Technology, Chongqing, China), and DBE (EN-450P5, Fujinon, Saitama, Japan) was carried out using an oral and/or anal approach. An enhanced CT of the abdomen and radiological findings were performed in some cases; however, the timing and cost of examination differed in each case.

The stool specimens were collected from all of the patients who had been hospitalized on the first day, and new containers were distributed to obtain new specimens per patient every morning. The stool samples were required to be fresh and not previously exposed or corrupted and brought to the laboratory within $10 \mathrm{hr}$. The examination of the stool and the accompanying eggs was routinely performed in our department; in parallel, repeated stool and egg examinations were needed when a physician ascertained that the patient was immunocompromised or had any of the following parasite-related conditions: occupation, home, behavior, or experience in an area where the parasites are known to have caused intestinal disease. Unpreserved stool samples were directly examined using wet mounts (saline and D'Antoni's iodine). Concentration wet mounts (saline and D'Antoni's iodine) and were then prepared with the formalin-ethyl acetate method [6]. The clinical information of each patient was retrieved from the clinical report of the referring doctor. In cases of missing data, the referring doctor was contacted via phone.

\section{RESULTS}

The final diagnoses of 424 overt OGIB patients are illustrated in Fig. 1. Overall, 31 patients with OGIB were diagnosed with a hookworm infection, a rate of 7.3\% (31/424). Hookworm infection is a known cause of overt OGIB. Upon examination, 17 of 227 and 14 out of 197 patients were diagnosed with hookworm infections in the CE and DBE groups, respectively. We found that 21 cases were older patients ( $>50$ years 


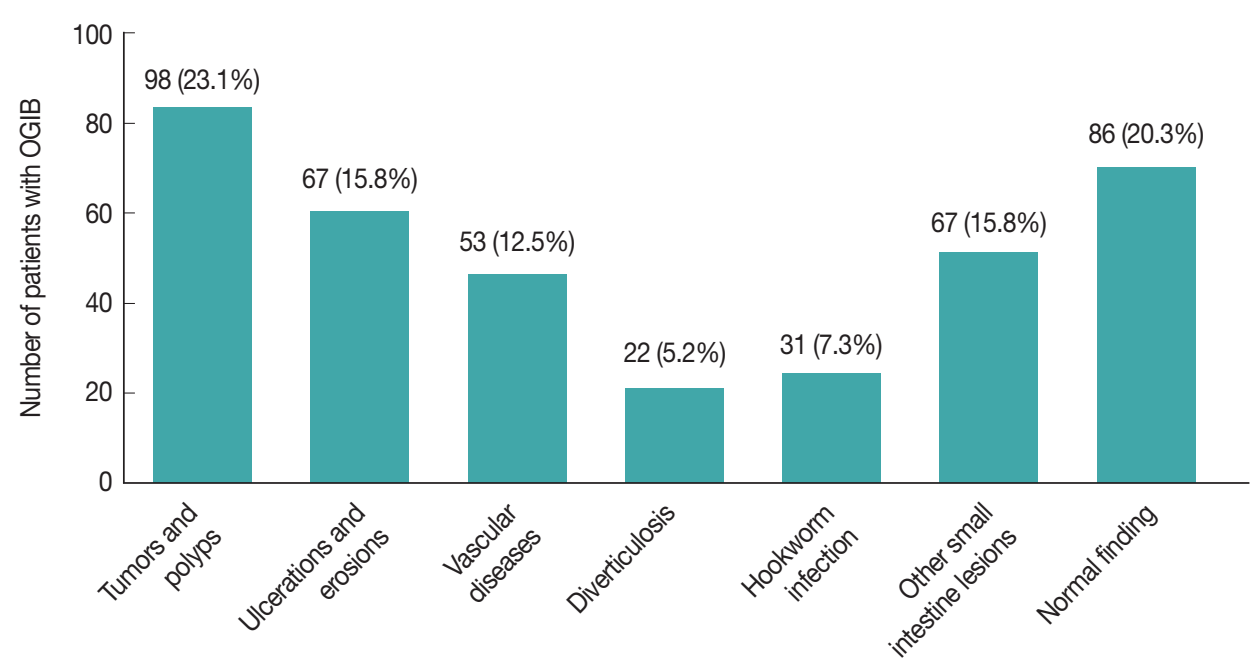

Fig. 1. Final diagnosis of cases with obscure gastrointestinal bleeding (OGIB). In total, 31 patients with overt OGIB were diagnosed with hookworm infections, a rate of $6.9 \%$.
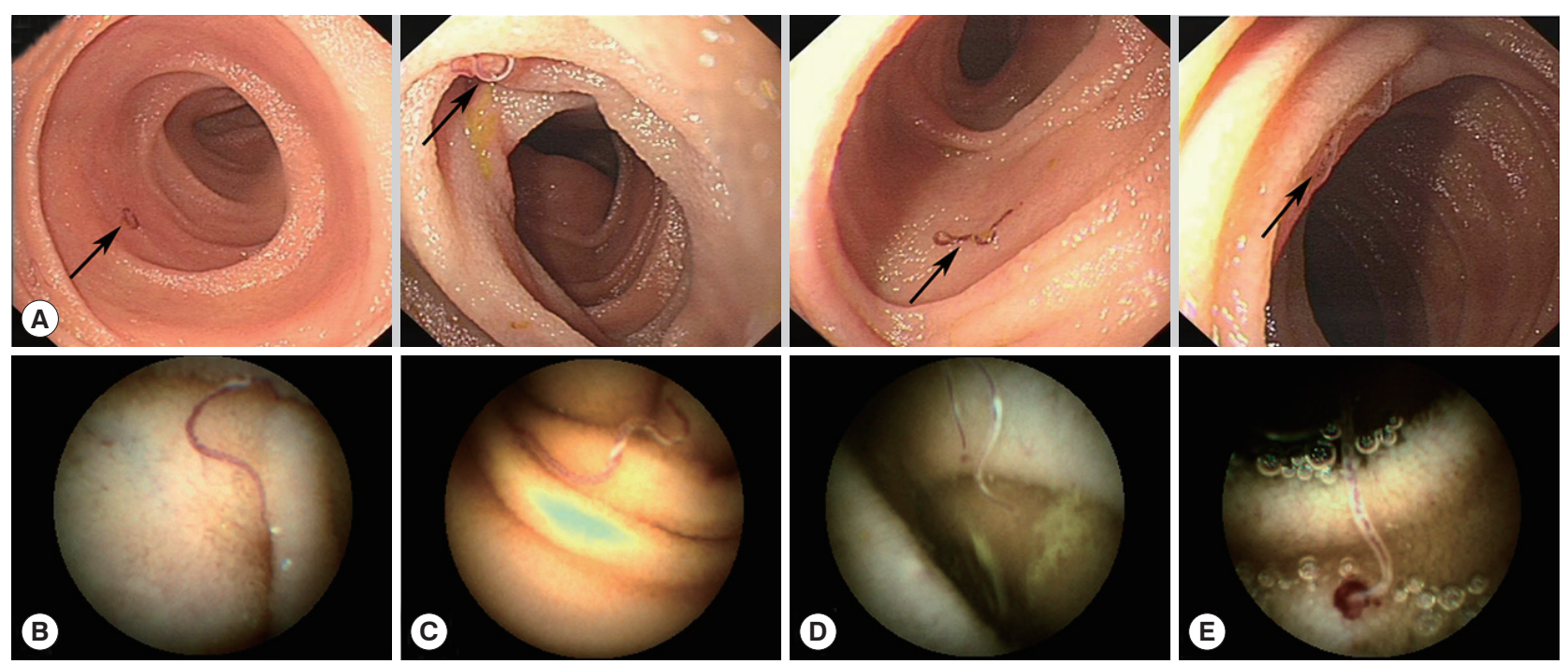

Fig. 2. Hookworm infections in the patient's small bowel, as observed with endoscopy. (A) Most hookworms frequently appeared between the distal duodenum and the distal jejunum. $(\mathrm{B}, \mathrm{C})$ Hookworms appeared with reddish bodies attached to the intestinal mucosa. (D) Hookworms feeding on the intestinal mucosa with a hemorrhagic spot. (E) Blood oozing was found at the hookworms' site of attachment.

old). Hookworm infections were found in 17 female patients and 14 male patients. All of the patients were from high-infection areas and were admitted to hospital. Upon admission, the vital signs of most cases were stable, and the physical examination showed no abnormalities except for pale conjunctivae and slight abdominal tenderness. The numerous blood tests performed are listed in Table 1. Blood counts showed a hemoglobin (Hb) range of 4.2 to $14.5 \mathrm{~g} / \mathrm{dl}$; a severe degree of anemia was found in 12 cases, of which red blood cell counts ranged from $1.95 \times 10^{12} / \mathrm{L}$ to $4.91 \times 10^{12} / \mathrm{L}$, and eosinophil counts ranged from $0.03 \%$ to $33.8 \%$. Fecal occult blood was found positive in 25 cases. The stool examination was positive for eggs or adult parasites in 4 cases. The electrolyte, liver and renal function tests were all normal, and the tumor markers were negative. Enhanced CT images of the abdomen and radiological findings were taken in some cases but were found to be nonspecific. Most of the cases assessed by EGD or colonoscopy showing hookworm infection-induced overt OGIB had nonspecific clinical manifestations.

Under performing DBE, we found several hookworms from 


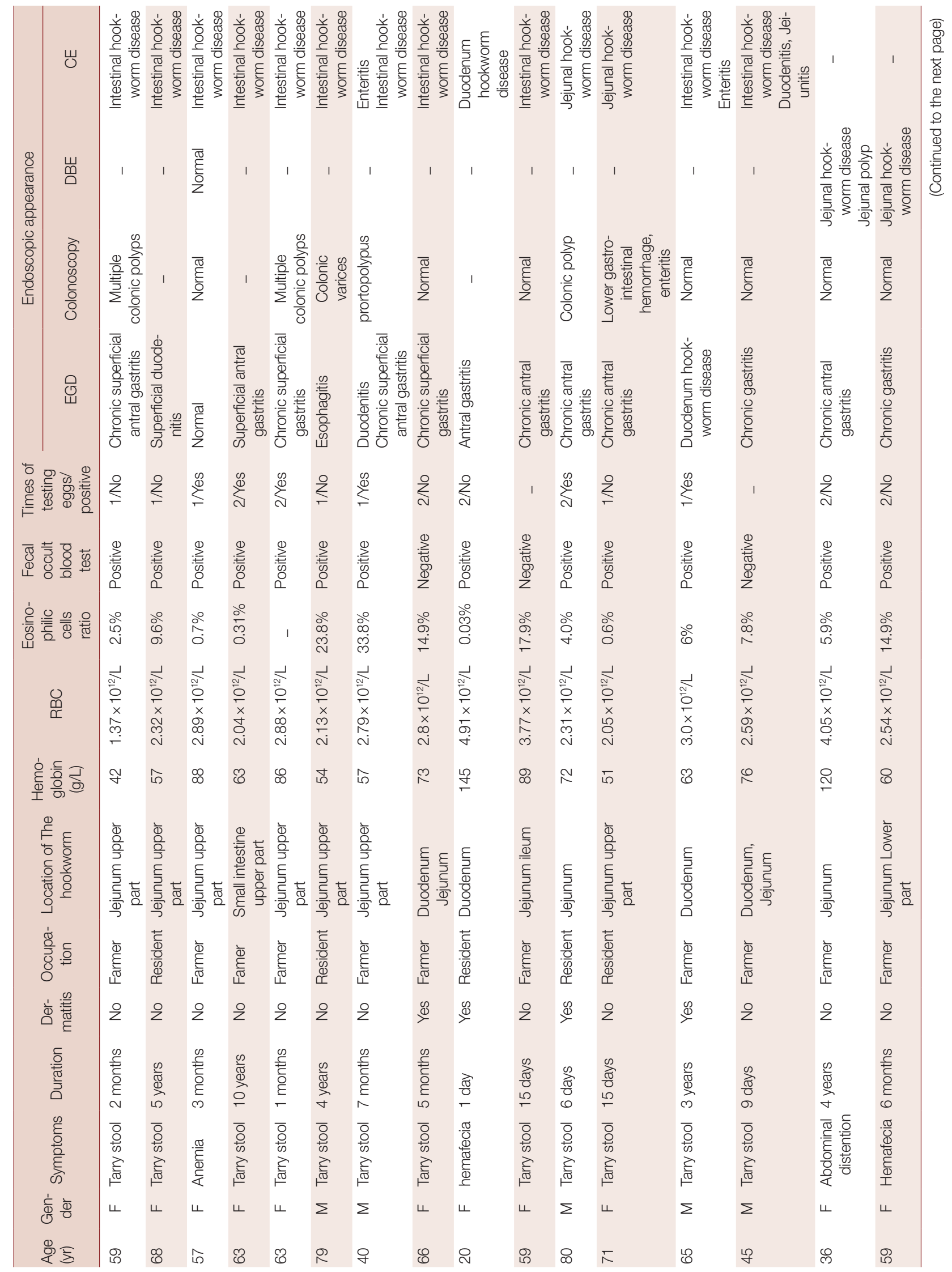




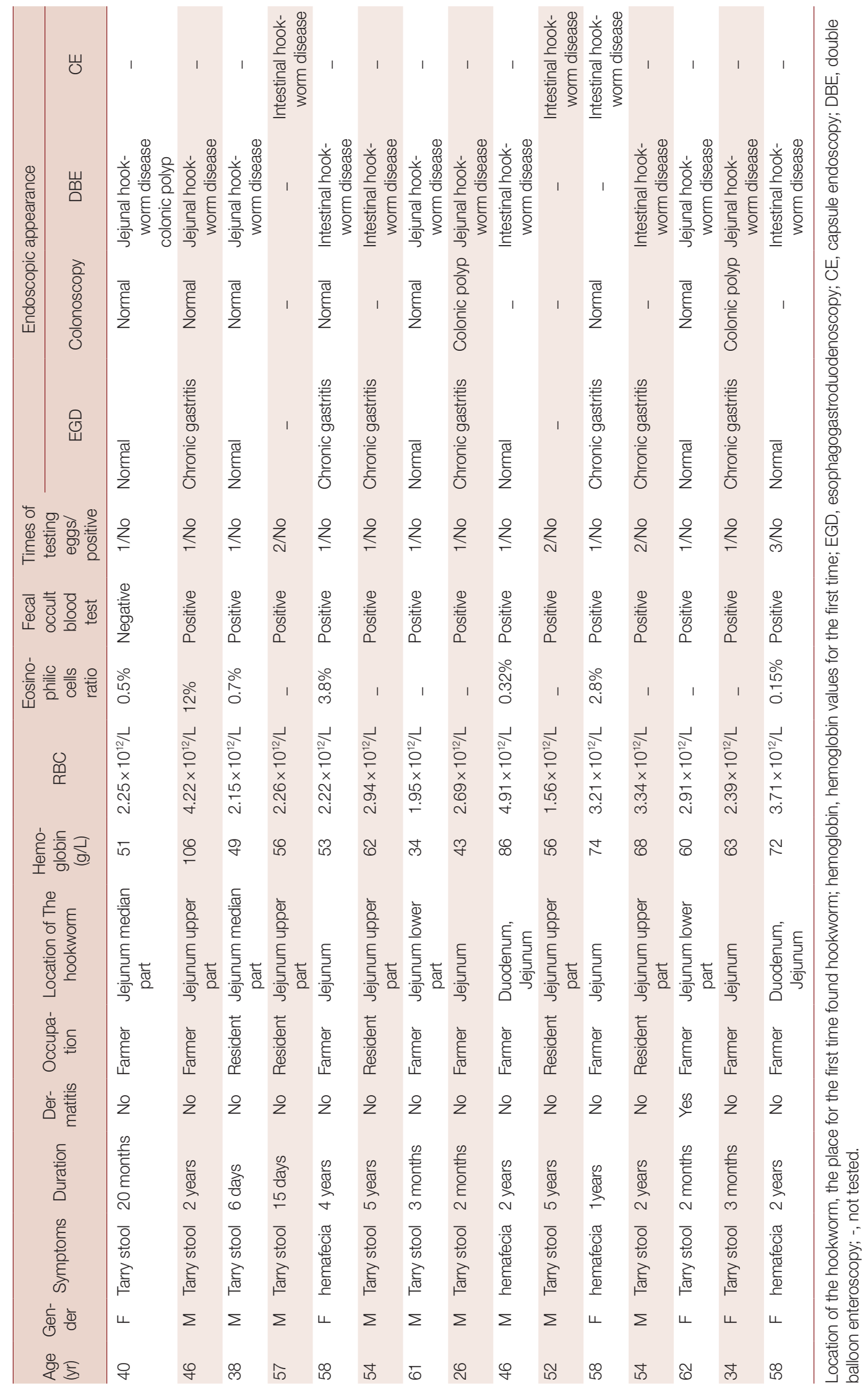




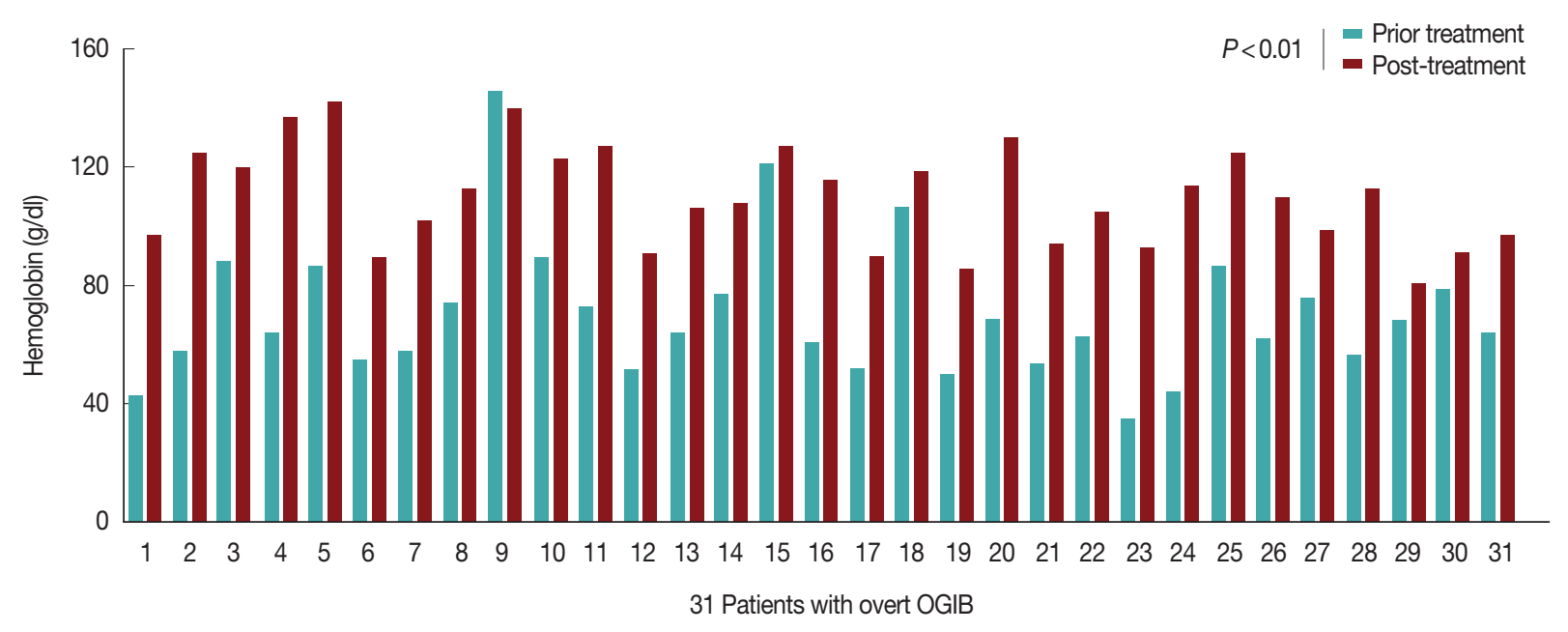

Fig. 3. The hemoglobin $(\mathrm{Hb})$ of 30 cases recovered after treatment compared to before treatment. The 31 patients did not experience any recurrent bleeding, and their $\mathrm{Hb}$ increased significantly throughout treatment after 6 months.

the distal duodenum to the distal jejunum in a single patient, as shown in Fig. 2A. The hookworms appeared as having reddish bodies attached to the intestinal mucosa (Fig. 2B, C). Hookworms feeding on the intestinal mucosa had a hemorrhagic spot at the point of their attachment to the mucosa (Fig. 2D). Oozing blood was found at the hookworm attachment site in some cases (Fig. 2E) under CE. The parasites were either withdrawing blood while attached to the mucosal surface of the intestine or they had previously attacked the areas and concomitant bleeding lesions were found.

No other concomitant bleeding disease was found in any of the 31 patients. The patients were treated with albendazole 400 mg twice a day for 3 days, and blood transfusions and iron replacements were administered. In the follow up, none of the 31 patients bled again, and after 6 months, their hemoglobin had increased significantly, as illustrated in Fig. 3. The stool eggs and adult parasite tests remained negative over the ensuing 3 months of follow up.

\section{DISCUSSION}

In poor tropical and subtropical areas, there are large numbers of patients with massive hookworm infestations, but very few of them show symptoms of overt bleeding from the GI tract, which is often ignored. Therefore, physicians should be cautious in attributing the cause of overt GI bleeds to hookworm infestations alone.

In our study, 31 patients with overt OGIB were diagnosed with hookworm infections, a rate of $7.3 \%$ (31/424). The mor- bidity of hookworm infection in the southwest region of China is higher than reported in other parts of the world [7]. This is likely related to the epidemiological characteristics in the region, where subsistence farming and animal husbandry are commonly practiced. Moreover, some families lack basic infrastructure, such as a supply of treated water at the household level or their own toilet. Based on the above observations, hookworm infections seem to be closely related to farmers working in the field, barefoot. Meanwhile, we found that the rate of hookworm infections was higher in females than in males. However, Peter J. Hotez reported that the overall prevalence and intensity of hookworm infections are higher in males than in females [4]. We believe that our results are due to the prevalence of the performance of farm labor in southwest China by women, who are therefore more often exposed to infection, while most of the men work in the city. Moreover, the intensity of infection usually reached a peak during childhood and adolescence in previously reported papers [8]. In contrast, there appears to be considerable variation in the age intensity profiles of hookworm infection, according to our study. Age is related to the rate of hookworm infection, with the highest intensity occurring in middle-aged individuals and those over the age of 80 . Therefore, we believe that the present infection patterns have implications for the world's expanding elderly population. Additionally, school-based deworming has become the largest public health program in China, as it offers a number of health-related benefits to children [9]. Third, hookworms are refractory to host immunity during the host's life, and the intensity of infection gradually rises with the age of 
the host [10].

The hookworm constantly changes the adsorption site and secretion of anticoagulant substances, resulting in wound blood loss is the main adverse outcome. Since the subtle but significant impact of hookworms on human's health was first recognized by scholars, international efforts to control the morbidity caused by this infection have been conducted [11]. According to our research, hookworms most frequently parasitize the proximal part of the small intestine, which can be observed in the attacked areas of the mucosa in Fig. 2. The 12 patients had severe anemia, and the lowest hemoglobin level reached $4.2 \mathrm{~g} /$ $\mathrm{dl}$, owing to the hookworms' sucking blood from the capillaries and arterioles. Hookworms also secrete Ancylostoma ceylanicum anticoagulant peptide-1, inhibiting the blood coagulation in the attachment site and leading to the loss of blood from the intestine [11]. In addition, they can carry antigens and pass through a range of host tissues, surviving within the host by warding off immune attacks [12]. The presence of more than 40 adult hookworms has been reported to be adequate to cause a host hemoglobin level of less than $6.0 \mathrm{~g} / \mathrm{dl}$ [12]. There have been at least 13 reported cases of acute hemorrhage caused by hookworm infection. And some hold that a hookworm burden (i.e., the intensity of infection or the number of worms per person) of at least 40-160 worms is associated with $\mathrm{Hb}$ levels below $11 \mathrm{~g} / \mathrm{dl}$ [13]. Moreover, this low-volume, slow oozing of blood is unlikely to cause severe melena, which leads to a significant drop in $\mathrm{Hb}$ [14]. We hypothesize that hookworms may damage the blood vessels of the small intestine for a long period of time, inducing acute massive intestinal bleeding; heavy burden individuals can lose up to $250 \mathrm{ml}$ of blood per day [15]. Our case with severe overt obscure gastrointestinal bleeding may have had the following contributing factors. First, the patient had mild chronic anemia after hookworm infection, and the overt GI bleeding led to severe visible blood loss, especially due to the inadequate initial iron stores of the host. Second, although the endoscopic investigation and stool microscopy of patients in endemic areas commonly result in a diagnosis of hookworms, these procedures are rarely repeated during the diagnostic process.

Another important marker is eosinophilia, which can be detected in $30 \%$ to $60 \%$ of cases [16]; its peak usually coincides with the development of adult hookworms in the intestine and occurs 5-9 weeks after the onset of the infection [17]. Hookworms can induce the production of cytokines, such as IL-4, IL-5, and parasite-specific immunoglobulin E, which lead to the expansion and mobilization of eosinophils [4].

The simple but important diagnosis of hookworm infestation is based on the microscopic examination of feces to detect hookworm eggs [6]. In our study, $0.85 \% \mathrm{NaCl}$ solution was used as the diluent, and stool microscopy identified eggs in 6 cases, as illustrated in Table 1. However, stool microscopy failed to identify any eggs in the rest of the cases. We are not sure if the color of the bloody or black stool can affect the detection of parasite eggs. In our stool examinations, there were 2 observations that require closer attention. First, the overall positivity rate was higher for a 3 specimen series than for a single specimen [5]. Moreover, some organisms, such as Strongyloides species, require more than 10 examinations as well as special procedures (Baermann technique and agar plate culture, for example), to ensure their final detection. Second, the responsibility for this categorization depends on detailed, communication between physicians and the laboratory to ensure that additional examinations of specimens are performed when needed. It has been reported that quantitative egg counts serve as an indirect measure of the adult-hookworm burden, in parallel to Hb levels [18].

The hookworms were almost always found in the proximal portion of the small intestine, that is, in the duodenum and the upper portion of the jejunum. Therefore, hookworm infections can also cause gastritis and duodenitis in some cases. Thus, repeated examinations of unexplained bleeding after endoscopy treatment are called for, and recurrent bleeding should be considered to be an indicator for continued vigilance for another diagnosis after a so-called "clear" diagnosis. Jejunal histologic examinations predominantly reveal eosinophilic inflammation, which is consistent with the histological changes in the small bowel under worm infestations. In the diagnostic process, we should focus on accompanying symptoms such as peptic ulcers without neglecting the primary disease.

In addition to the digestive tract symptoms caused by hookworms, other systemic diseases can arise. Four of the present cases had dermatitis. Cases with A. duodenale third-stage larvae experience a ground itch, a local papular rash and erythematous, accompanied by pruritus on the feet and hands. The skin becomes waxy and turns a sickly yellowish color due to the penetrating larvae [19]. When the hookworms' third-stage larvae invade the skin, pass through the vasculature and enter the lungs, pneumonitis can result [18]. The oral ingestion of duodenale larvae can result in Wakana syndrome, which is characterized by nausea, vomiting, pharyngeal irritation, cough, dys- 
pnea, and hoarseness. The chronic protein loss from patients with heavy hookworm infections can result in hypoproteinemia or anasarca [19].

In summary, there are sporadic case reports of hookworm infections in overt OGIB. CE and DBE are currently the preferred tests for the evaluation of a hookworm infection in the entire small intestine and should be part of the initial diagnosis in patients with overt OGIB. In our study, we found that most of these cases occurred in hookworm-endemic areas [14, 20]; the physician must overcome the tendency to ignore the symptoms of hookworms, lest he misdiagnose the patient. Specially, combined multiple stool tests and multiple stool samplings can greatly improve the rate of successful hookworm diagnoses. As the testing algorithm is customized for the patient through the application of clinical judgment, good communication between doctors and patients is essential to prevent the need for more urgent treatments and lower the overall healthcare utilization and costs.

\section{CONFLICT OF INTEREST}

Kun-Yan Wei, Qiong Yan, Bo Tang, Shi-Ming Yang, Peng-Bing Zhang, Ming-Ming Deng, and Mu-Han Lü have no conflicts of interest or financial ties to disclose.

\section{REFERENCES}

1. Raju GS, Gerson L, Das A, Lewis B. American Gastroenterological Association (AGA) Institute medical position statement on obscure gastrointestinal bleeding. Gastroenterology 2007; 133: 1694-1696.

2. American Gastroenterological Association Medical Position Statement: guidelines on intestinal ischemia. Gastroenterology 2000; 118: 951-953.

3. Steinmann P, Du ZW, Wang LB, Wang XZ, Jiang JY, Li LH, Marti $\mathrm{H}$, Zhou XN, Utzinger J. Extensive multiparasitism in a village of Yunnan province, People's Republic of China, revealed by a suite of diagnostic methods. Am J Trop Med Hyg 2008; 78: 760-769.

4. Liao Z, Gao R, Li F, Xu C, Zhou Y, Wang JS, Li ZS. Fields of applications, diagnostic yields and findings of OMOM capsule endoscopy in 2400 Chinese patients. World J Gastroenterol 2010; 16 :
2669-2676.

5. Lintermans JP. Severe intestinal bleeding leading to exploratory laparotomy in an infant with hookworm infection. Clin Pediatr (Phila) 1976; 15: 1073-1074.

6. Rosenblatt JE. Clinical importance of adequately performed stool ova and parasite examinations. Clin Infect Dis 2006; 42: 979-980.

7. Maxwell C, Hussain R, Nutman TB, Poindexter RW, Little MD, Schad GA, Ottesen EA. The clinical and immunologic responses of normal human volunteers to low dose hookworm (Necator americanus) infection. Am J Trop Med Hyg 1987; 37: 126-134.

8. Goenka MK, Majumder S, Kumar S, Sethy PK, Goenka U. Single center experience of capsule endoscopy in patients with obscure gastrointestinal bleeding. World J Gastroenterol 2011; 17: 774778.

9. Budhathoki S, Shah D, Bhurtyal KK, Amatya R, Dutta AK. Hookworm causing melaena and severe anaemia in early infancy. Ann Trop Paediatr 2008; 28: 293-296.

10. Zheng Q, Chen Y, Zhang HB, Chen JX, Zhou XN. The control of hookworm infection in China. Parasit Vectors 2009; 2: 44.

11. Diemert DJ, Bethony JM, Hotez PJ. Hookworm vaccines. Clin Infect Dis 2008; 46: 282-288.

12. Hotez PJ, Bethony J, Bottazzi ME, Brooker S, Buss P. Hookworm: "the great infection of mankind". PLoS Med 2005; 2: e67.

13. Kelley PW, Takafuji ET, Wiener H, Milhous W, Miller R, Thompson NJ, Schantz P, Miller RN. An outbreak of hookworm infection associated with military operations in Grenada. Mil Med 1989; 154: 55-59.

14. Chen YY, Soon MS. Endoscopic diagnosis of hookworm infection that caused intestinal bleeding. Gastrointest Endosc 2005; 62: 142.

15. Roche M, Perez-Gimenez ME, Layrisse M, Di Prisco ME. Study of urinary and fecal excretion of radioactive chromium Cr51 in man; its use in the measurement of intestinal blood loss associated with hookworm infection. J Clin Invest 1957; 36: 1183-1192.

16. Brooker S, Bethony J, Hotez PJ. Human hookworm infection in the 21st century. Adv Parasitol 2004; 58: 197-288.

17. de Silva NR, Brooker S, Hotez PJ, Montresor A, Engels D, Savioli L. Soil-transmitted helminth infections: updating the global picture. Trends Parasitol 2003; 19: 547-551.

18. Hotez PJ, Brooker S, Bethony JM, Bottazzi ME, Loukas A, Xiao S. Hookworm infection. N Engl J Med 2004; 351: 799-807.

19. Branda JA, Lin TY, Rosenberg ES, Halpern EF, Ferraro MJ. A rational approach to the stool ova and parasite examination. Clin Infect Dis 2006; 42: 972-978.

20. Hochedez P, Caumes E. Hookworm-related cutaneous larva migrans. J Travel Med 2007; 14: 326-333. 